\title{
Stabilization Study of Modified PAN- co-Polymer Composite Nanofibers with Functionalized Single-Walled Carbon Nanotubes
}

\author{
Fateme Farhadi ${ }^{1}$, Marjan Abbasi ${ }^{1}$, and Akbar Khodaparast Haghi ${ }^{1}$ \\ ${ }^{1}$ University of Guilan
}

June 29, 2020

\begin{abstract}
In the present study, polyacrylonitrile (PAN)-co-polymer nanofibers as well as PAN-co-polymer nanofibers reinforced with functionalized single - walled carbon nanotubes (F-SWCNTs) were produced by electrospinning and stabilized. The samples were evaluated using DSC, FTIR, SEM and XRD. In the sample containing F-SWCNT the amount of heat released during the stabilization reactions was lower than that of pure PAN nanofibers. This indicates that the F-SWCNT prevents the sudden release of heat and damage to the nanofibers during stabilization. The carbon nanotubes greatly prevent the reduction of the diameter of the nanofibers as well as the decrease in the size of the crystals and the decrease of the arrangement of the nanofibers during stabilization.
\end{abstract}

\section{Hosted file}

pol.20200431.pdf available at https://authorea.com/users/337827/articles/463391stabilization-study-of-modified-pan-co-polymer-composite-nanofibers-with-functionalizedsingle-walled-carbon-nanotubes 